\title{
Team Performance with Test Scores
}

\author{
JON KLEINBERG and MAITHRA RAGHU, Cornell University
}

\begin{abstract}
Team performance is a ubiquitous area of inquiry in the social sciences, and it motivates the problem of team selection-choosing the members of a team for maximum performance. Influential work of Hong and Page has argued that testing individuals in isolation and then assembling the highest scoring ones into a team is not an effective method for team selection. For a broad class of performance measures, based on the expected maximum of random variables representing individual candidates, we show that tests directly measuring individual performance are indeed ineffective, but that a more subtle family of tests used in isolation can provide a constant-factor approximation for team performance. These new tests measure the "potential" of individuals, in a precise sense, rather than performance; to our knowledge they represent the first time that individual tests have been shown to produce near-optimal teams for a nontrivial team performance measure. We also show families of subdmodular and supermodular team performance functions for which no test applied to individuals can produce near-optimal teams, and we discuss implications for submodular maximization via hill-climbing.
\end{abstract}

CCS Concepts: • Mathematics of computing $\rightarrow$ Probabilistic algorithms; $\bullet$ Theory of computation $\rightarrow$ Social networks;

Additional Key Words and Phrases: Algorithms, learning and game theory

ACM Reference format:

Jon Kleinberg and Maithra Raghu. 2018. Team Performance with Test Scores. ACM Trans. Econ. Comput. 6, 3-4, Article 17 (October 2018), 26 pages.

https://doi.org/10.1145/3274644

\section{INTRODUCTION}

The performance of teams in solving problems has been a subject of considerable interest in multiple areas of the mathematical social sciences [9, 14, 23]. The ways in which groups of people come together and accomplish tasks is an important issue in theories of organizations, innovation, and other collective phenomena, and the recent growth of interest in crowdwork has brought these issues into focus for online platforms as well.

In formal models of team performance, a central issue is the problem of team selection. Suppose there is a task to be accomplished and we can assemble a team to collectively work on this task, drawing team members from a large set $U$ of $n$ candidates. (We can think of $U$ as the job applicants

This work was supported in part by a Simons Investigator Award, a Google Research Grant, a Facebook Faculty Research Grant, an ARO MURI grant, and NSF grant IIS-0910664.

Authors' addresses: J. Kleinberg and M. Raghu, Cornell University, Ithaca, NY; emails: \{kleinber, mraghu\}@cs.cornell. edu.

Permission to make digital or hard copies of all or part of this work for personal or classroom use is granted without fee provided that copies are not made or distributed for profit or commercial advantage and that copies bear this notice and the full citation on the first page. Copyrights for components of this work owned by others than ACM must be honored. Abstracting with credit is permitted. To copy otherwise, or republish, to post on servers or to redistribute to lists, requires prior specific permission and/or a fee. Request permissions from permissions@acm.org.

(C) 2018 Association for Computing Machinery.

2167-8375/2018/10-ART17 \$15.00

https://doi.org/10.1145/3274644 
for this task.) A team can be any subset $T \subseteq U$, and its performance in collectively working on the task is given by a set function $g(T)$. The central optimization problem is therefore a kind of set function maximization: Given a target size $k<n$ for the team, we would like to find a set $T$ of cardinality $k$ for which $g(T)$ is as large as possible.

The generality of this framework has meant that it can be used to reason about a wide range of settings in which we hire workers, solicit advice from a committee, run a crowdsourced contest, admit college applicants, and many other activities-all cases where we have an objective function (the outcome of the work performed, the quality of the insights obtained, or reputation of the group that is assembled) that is a function of the set of people we bring together.

Models of Team Performance. Different models of team performance can be interpreted as positing different forms for the structure of the set function $g(\cdot)$. Some of the most prominent have been the following:

- Cumulative effects. Arguably the simplest team performance function is a linear one: Each individual can produce work at a certain volume, and the team's performance is simply the sum of these individual outputs. Formally, we assume that each individual $i \in U$ has a weight $w_{i}$, and then $g(T)=\sum_{i \in T} w_{i}$.

- Contests. Much work has focused on models of team performance in which the "team" is highly decoupled: Members attempt the task independently, and the quality of the outcome is the maximum quality produced by any member. Such formalisms arise in the study of contest-like processes, where many competitors independently contribute proposed solutions, and a coordinator selects the best one (or perhaps the $h$ best for some $h<k$ ) $[13,15]$. Note, however, that this objective function is applicable more generally to any setting with a "contest structure," even potentially inside a single organization, where proposed solutions are generated independently and the outcome is judged by the quality of the best one (or best few). It can also apply to a group whose reputation is judged on the maximum future achievement of any of its members; for example, one could imagine an admissions committee trying to select a group of $k$ top applicants, with the goal of optimizing the maximum future success of any of them.

- Complementarity. Related to contests are models in which each team member has a set of "perspectives," and the quality of the team's performance grows with the number of distinct perspectives that they are collectively able to provide [11, 17].

- Synergy. In a different direction, research has also considered models of team performance in which interaction is important, using objective functions with terms that generate value from pairwise interaction between team members [1].

These settings are not just different in their motivation; they rely on functions $g(\cdot)$ with genuinely different combinatorial properties. In particular, in the language of set functions, the first class of instances is based on modular (i.e., linear) functions, the second and third classes are based on submodular functions, and the fourth is based on supermodular functions.

The second and third classes of functions-contests and complementarity-play a central role in Scott Page's highly influential line of work on the power of diversity in team performance [20]. The argument, in essence, is that a group with diversity that is reflected in independent solutions or complementary perspectives can often outperform a group of high-achieving but like-minded members.

Evaluating Team Members via Tests. A key issue that Page's work brings to the fore is the question of tests and their effectiveness in identifying good team members [20]. In most settings, one can't "preview" the behavior of a set of team members together, and so a fundamental approach 
to team formation is to give each candidate $i \in U$ a test, resulting in a test score $f(i)$ [18]. It is natural to then select the $k$ candidates with the highest test scores, resulting in a team $T$. We could think of the test score $f(i)$ corresponding to the SAT or GRE score in the case of college or graduate school admissions, or corresponding to the quality of answers to a set of technical interview questions in a job interview. We note that this issue of tests as a method of selection is a contribution of Page's work that is related to the issue of diversity, but it also has interesting implications independently of diversity, and it is the properties of tests that serves as our focus in the present article.

Should we expect that the $k$ individuals who score highest on the test will indeed make the best team? In a simple enough setting, the answer is yes: For modular functions $g(T)=\sum_{i \in T} w_{i}$, it is enough to evaluate each candidate $i$ in isolation, applying the test $f(i)=g(\{i\})=w_{i}$. Let us refer to $f(i)=g(\{i\})$ in general as the canonical test: We simply see how $i$ would perform as a one-element set. For modular functions, clearly the $k$ candidates with the highest scores under the canonical test form the best team.

On the other hand, Hong and Page construct an example based on complementarity, in which the $k$ candidates who score highest on the canonical test perform significantly worse as a team than a set of $k$ randomly selected candidates [11]. Their mathematical analysis has a natural interpretation with implications for hiring and admissions processes: The $k$ candidates who score highest on the test are too similar to each other, and so, with an objective function based on complementarity, they collectively represent many fewer perspectives than a random set of $k$ candidates.

Beyond these compelling examples, however, there is very little broader theoretical understanding of the power of tests in selecting teams. Thinking of tests as arbitrary functions of the candidates is not a perspective that has been present in this earlier work; a particularly unexplored issue is the fact that the failure of the canonical test doesn't necessarily rule out the possibility that other tests might be effective in assembling teams. Does it ever help, in a formal sense, to evaluate a candidate using a measure $f(i)$ that is different from his or her actual individual performance at the task? In real settings, we see many cases where employers, search committees, or admissions committees evaluate applicants on their "potential" rather than on their demonstrated performance; is this simply a practice that has evolved for reasons of its own, or does it have a reflection in a formal model of team selection? Without a general formulation of tests as a means for evaluating team members, it is difficult to offer insights into these basic questions.

The Present Work: Effective Tests for Team Selection. In this article, we analyze the power of general tests in forming teams across a range of models. Our main result is the finding that, for team performance measures that have a contest structure, near-optimal teams can be selected by giving each candidate a test in isolation and then ranking by test scores, but only using tests that are quite different from the canonical test. To our knowledge, this is the first result to establish that nonstandard tests can yield good team performance in settings where the canonical test provably fails.

In more detail, in a contest structure, each candidate $i \in U$ has an associated discrete random variable $X_{i}$, with all random variables mutually independent, and the performance of a team $T \subseteq U$ is the expected value of the random variable $\max _{i \in T} X_{i}$. More generally, we may care about the top $h$ values, for a parameter $h<k$, in which case the performance of $T$ is the expected value of the sum of the $h$ largest random variables in $T$ :

$$
g(T)=E\left[\max _{S \subseteq T,|S|=h} \sum_{i \in S} X_{i}\right] .
$$


The test that works well for these contest functions has a natural and appealing interpretation. Focusing on the general case with parameter $h<k$, we define the test score $f(i)$ to be

$$
E\left[\max \left(X_{i}^{(1)}, X_{i}^{(2)}, \ldots, X_{i}^{(k / h)}\right)\right]
$$

where $X_{i}^{(1)}, X_{i}^{(2)}, \ldots, X_{i}^{(k / h)}$ represent $k / h$ independent random variables all with the same distribution as $X_{i}$.

The fact that this test works for assembling near-optimal teams in our contest setting has a striking interpretation: It provides a formalization of the idea that we should indeed sometimes evaluate candidates on their potential rather than on their demonstrated performance. Indeed, $\max \left(X_{i}^{(1)}, X_{i}^{(2)}, \ldots, X_{i}^{(k / h)}\right)$ is precisely a measure of potential, since, instead of just evaluating $i$ 's expected performance $E\left[X_{i}\right]$, we're instead asking, "If $i$ were allowed to attempt the task $k / h$ times independently, what would the best-case outcome look like?" Like the argument of Hong and Page about diversity, this argument about potential has qualitative implications for evaluating candidates in certain settings, implying that we should think about upside potential using a thought experiment in which candidates are allowed multiple independent tries at a task.

Following this result, we then prove a number of other theorems that help round out the picture of general tests and their power. We first show a closely related test that also provides a method for constructing near-optimal teams in which $f(i)$ is defined to be the conditional expectation of $X_{i}$, conditioned on its taking a value in the top $(1 / k)$ fraction of its distribution. We also show that there exists an absolute constant $c>1$ such that no test can construct teams under our objective function with performance guaranteed to come within a factor $c$ of optimal.

Next, we show that there are natural objective functions for which no test can yield near-optimal results for team selection; these include certain submodular functions capturing complementarity and certain supermodular functions representing synergy. Note that this is a much stronger statement than simply asserting the failure of the canonical test since it says that no test can produce near-optimal teams. Finally, we identify some further respects in which team performance functions $g(\cdot)$ based on contest structures have tractable properties, in particular showing that, for the special case in which the random variables corresponding to all the candidates are weighted Bernoulli variables, greedy hill-climbing on the value of $g(\cdot)$ in fact produces an exactly optimal set of size $k$.

The Power of Tests in Competitive Settings. Our discussion of test scores can be viewed as pursuing a family of questions of the following general form: "When evaluating the effectiveness of an individual, to what extent can we perform this evaluation in isolation, and to what extent do we need the context in which they are operating?"

This type of question can be asked in settings other than team formation, and, in the final section, we show how it leads to interesting results if we ask it in a setting with competition between individuals. Specifically, suppose we have a collection of competitors, and these competitors will be matched up in pairwise competitions. Each competitor $i$ is represented by a random variable $X_{i}$, representing the distribution of performance quality that $i$ exhibits in competition. When $i$ and $j$ are paired in a competition, we imagine that they draw values independently from $X_{i}$ and $X_{j}$, respectively, and the competitor who draws the larger value wins. (We'll say that they tie if the values drawn are equal.) Thus the probability that $X_{i}$ wins or ties is $\mathbb{P}\left(X_{i} \geq X_{j}\right)$.

We'd like to assign each competitor with random variable $X$ a score $f(X)$, based only on $X$ and not any of the other random variables, so that when two competitors are paired up, the one with the higher score has a reasonably large probability of winning (or tieing). In other words, we'd like to find a function $f$ defined on arbitrary random variables, and an absolute constant $c>0$, such that if $f(X) \geq f(Y)$, then $\mathbb{P}(X \geq Y) \geq c$. 
Is this possible, and, if so, how large can we make $c$ ? We give a tight answer to this question: The largest possible $c$ is $c=1 / 4$. To do this, we first establish that $c=1 / 4$ can be achieved by the function $f$ that maps each $X$ to its median $f(X)$. We then establish that $c$ cannot be any larger using an argument based on the notion of nontransitive dice.

We feel that the emergence of rich questions in this very different domain suggests that there may be other unexpected settings in which an understanding of test scores might lead to interesting insights.

\section{TEAM SELECTION BY TEST SCORE}

In this section, we formalize our goal of picking individuals via a test score to maximize a notion of team performance. We precisely define our measure of team performance and also define a test that can be applied to individuals for team selections. This test is particularly remarkable because no matter the size of the team we pick using this test, we can give a constant (independent of team size) order performance guarantee on our test-selected team compared to the optimal team. The latter parts of this section build the necessary mathematical tools and definitions needed and then prove this result.

In doing so, we build on basic properties of the maximum over sets of random variables and expect that these results will be useful more broadly.

\subsection{Problem Setting and Key Definitions}

Suppose we are trying to assemble a team of fixed size $k$. We have $N$ possible candidates for this team, each associated with a non-negative discrete random variable $X_{i}$. Each $X_{i}$ represents the latent ability of the candidate. For example, if $X_{i}$ took values $(1,0.4,0)$ with probabilities $(0.75,0.2,0.05)$, candidate $i$, when put to test, will most likely (with probability 0.75$)$ perform with skill 1 and, with lower chance (probability 0.2 ), perform with skill 0.4 . There is also a small chance (probability 0.05 ) that they might perform very poorly, with skill 0 . Setting up notation, we assume each $X_{i}$ has a distribution $\left(p_{1}, \ldots, p_{n}\right)$ over non-negative values $\left(x_{1}, \ldots, x_{n}\right)$, with $x_{1}>x_{2}, \ldots>x_{n} \geq 0$.

To select our team, we can test any of our candidates individually but not as a group. Testing a candidate individually corresponds to applying a scoring function $f\left(X_{i}\right)$ to the random variable $X_{i}$ representing the candidate. We can then rank candidates according to their scores and pick the top $k$ to form our team. The performance of our team is measured by a team scoring function $g$.

Our work first looks at devising a test function $f$ when the team scoring function $g$ is the expected maximum. Having picked our team to comprise of $X_{1}, \ldots, X_{k}$, the team performance is given by

$$
g\left(X_{1}, \ldots, X_{k}\right)=\mathbb{E}\left(\max \left\{X_{1}, \ldots, X_{k}\right\}\right)
$$

If the team scoring function is the expected maximum, an immediate first candidate for $f$ might be the expectation, $f\left(X_{i}\right)=\mathbb{E}\left(X_{i}\right)$, which we refer to as the canonical test. However, as discussed in Section 3, this first choice is highly suboptimal: We can show that picking a team according to this test results in a multiplicative factor $k$ performance difference between the chosen team and the optimal team. Instead, we define the following, more subtle test. Let $X^{(i)}$ be independent and identically distributed (iid) copies of the random variable $X$. Then:

$$
f(X)=\mathbb{E}\left(\max \left(X^{(1)}, \ldots, X^{(k)}\right)\right)
$$

We can interpret $f$ as a better test of the potential of $X$, where, instead of taking the expectation, we take the best effort when $X$ is given multiple $(k)$ attempts. Remarkably, picking a team according to this test results in a constant factor (independent of $k$ ) guarantee on the chosen team's performance compared to the optimal team. 
In the following subsections, we build toward and culminate with a proof of this result. In fact, we work with a more general individual test function $f$ and team scoring function $g$ :

Definition 2.1 (Team Performance Scoring Function). For (non-negative) random variables $X_{1}, \ldots, X_{k}$, and for $i \leq k$, let $X_{\left(X_{1}, \ldots, X_{k}\right)}^{(i)}$ denote the $i$ th largest random variable out of $X_{1}, \ldots, X_{k}$. Then, for $1 \leq h \leq k$, let:

$$
g_{h}\left(X_{1}, \ldots, X_{k}\right)=\mathbb{E}\left(X_{\left(X_{1}, \ldots, X_{k}\right)}^{(1)}+X_{\left(X_{1}, \ldots, X_{k}\right)}^{(2)}+\cdots+X_{\left(X_{1}, \ldots, X_{k}\right)}^{(h)}\right)
$$

Definition 2.2 (Individual Testing Function). For a non-negative discrete random variable $X$ and $h \leq k$, let

$$
f_{h}(X)=\mathbb{E}\left(\max \left(X^{(1)}, \ldots, X^{\left(\frac{k}{h}\right)}\right)\right)
$$

where $X^{(i)}$ denotes an iid copy of $X$.

These definitions provide a natural interpolation between potential and expected performance. For $h=1$, the team performance function $g$ again becomes the expected maximum, and, similarly, the individual scoring function $f$ is the corresponding "potential" test function defined earlier. Recall that, in this setting, the canonical test (the test of expected performance) is a very poor test for assembling a team. However, for $h=k$, the team performance function becomes $\mathbb{E}\left(\sum X_{i}\right)$, and the individual testing function collapses to the canonical test $\mathbb{E}\left(X_{i}\right)$. But as $\mathbb{E}\left(\sum X_{i}\right)=\sum \mathbb{E}\left(X_{i}\right)$, the canonical test is in this case the perfect test.

\subsection{Preliminary Mathematical Results: The Top $h / 2 k$ Quantile}

In the previous section, we defined our general team performance scoring function (for $h=1$, the expected maximum and more generally the expectation of the sum of the top $h$ performances of our team of size $k$ ) and our corresponding individual test function (for $h=1$ the expected maximum of $k$ copies of $X$ and more generally the expectation of the sum of the top $h$ performances of $k$ copies of $X$ ).

In this section, we derive important definitions and lemmas to allow us to prove the central result relating team performance when selecting with our test function: the constant factor performance guarantee with respect to the optimal team. Central to all of these is the notion of the top quantile of a random variable's distribution. Intuitively speaking, for some proportion $t$, we can define the top $t$ quantile of a discrete random variable to be the largest values taken by the random variable that are responsible for proportion $t$ of its probability mass. Returning to our example of $X_{i}$ with values $(1,0.4,0)$ and probabilities $(0.75,0.2,0.05)$, the top 0.6 quantile of $X_{i}$ would be $\{1\}$, as $X_{i}$ takes value 1 with probability $>0.6$. The top 0.8 quantile of $X_{i}$ would be $\{1,0.4\}$, as the probability mass of 1 alone is less than 0.8 , but the probability mass of both values combined is $>0.8$.

To formalize this, we turn to the notion of a random variable's sample space, treating our random variable $X$ as a function on events $\omega \in[0,1]$. We formalize this in the definition below. ${ }^{1}$

\footnotetext{
${ }^{1}$ We note that some of our basic definitions can be expressed in the language of order statistics, in which we take a set of given random variables $X_{1}, \ldots, X_{n}$ and a parameter $k$, and we construct a new random variable equal to the $k$ th largest value among $X_{1}, \ldots, X_{n}$ [5]. However, for our purposes, the general results about order statistics do not seem to provide more direct ways of handling any of the constructs in our analysis, and so we instead use the presentation developed in this section.
} 
Definition 2.3. For a non-negative discrete random variable $X$, we define, for $\omega \in[0,1]$,

$$
X(\omega)=\left\{\begin{array}{l}
x_{1} \text { if } \omega>1-p_{1} \\
x_{l} \text { if } 1-\sum_{i=1}^{l} p_{i}<\omega \leq 1-\sum_{i=l-1}^{n} p_{i} . \\
0 \text { if } \omega \leq 1-\sum_{i=1}^{n} p_{i}
\end{array}\right.
$$

With this definition, we can also make precise what we mean by the top values of $X$ :

Definition 2.4. For non-negative discrete $X$ with sample space $[0,1]$, the event $A$ that $X$ takes values in its top $h / 2 k$ quantile is

$$
A=\left\{\omega: \omega>1-\frac{h}{2 k}\right\}
$$

The top values of $X$ are then

$$
\left\{x_{i}: 1 \leq i \leq n, \exists \omega \in A, X(\omega)=x_{i}\right\} .
$$

Similarly, we can define the tail values to be

$$
\left\{x_{i}: 1 \leq i \leq n, \exists \omega \in A^{c}, X(\omega)=x_{i}\right\} .
$$

Returning to our example, if $h / 2 k=0.6$, then the top values of $X$ would be $\{1\}$, and the tail values would be $\{1,0.4,0\}$. If $h / 2 k=0.4$, then the top values would be $\{1,0.4\}$, and the tail values $\{0.4,0\}$. Note that there are values that appear in both top and tail in both the top and tail values, and indeed more generally, that the top values and tail values are usually not disjoint: For the boundary value $x_{t}$, we may have to split $\left\{\omega: X(\omega)=x_{t}\right\}$ into $A$ and $A^{c}$.

Before proceeding with the lemmas, we make a short comment on notation: From now on, all random variables $X$ are assumed to be discrete and non-negative, with probabilities $\left(p_{1}, \ldots, p_{n}\right)$ over values (in decreasing order) $\left(x_{1}, \ldots, x_{n}\right)$. We define $q_{i}$ to be the cumulative sum of the top $i$ probabilities; that is,

$$
q_{i}=\sum_{l=1}^{i} p_{l} .
$$

We will also often use $\left(x_{1}, \ldots, x_{t}\right)$ to denote the top values of $X$, with the probability mass associated with $x_{t}$ split so that $q_{t}=\frac{h}{2 k}$ exactly.

Our first two lemmas rely on the explicit form of our testing function $f_{h}$. In particular, with the definition of $q_{i}$, we have:

$$
\begin{aligned}
f_{h}(X)= & \left(\left(1-\left(1-q_{1}\right)^{k / h}\right) x_{1}+\left(\left(1-q_{1}\right)^{k / h}-\left(1-q_{2}\right)^{k / h}\right) x_{2}\right. \\
& \left.+\cdots+\left(\left(1-q_{n-1}\right)^{k / h}-\left(1-q_{n}\right)^{k / h}\right) x_{n}\right) .
\end{aligned}
$$

In the first two lemmas, we (i) bound the proportion that the top $h / 2 k$ quantile contributes to $f_{h}(X)$, and (ii) upper bound the contribution of the tail values of $X$ to $f_{h}(X)$. Splitting according to the top $h / 2 k$ is important because, for the main result, we bound $g_{h}$ by $f_{h}$ by evaluating the top and tail contributions separately.

Lemma 2.5. Let $X$ be a random variable, with underlying sample space $[0,1]$. Define $X^{\prime}$ as

$$
X^{\prime}(\omega)= \begin{cases}X(\omega) & \text { if } \omega>1-\frac{h}{2 k} \\ 0 & \mathrm{o} / \mathrm{w}\end{cases}
$$

Then

$$
f_{h}\left(X^{\prime}\right) \geq f_{h}(X)\left(1-\frac{1}{\sqrt{e}}\right) .
$$


Proof. First note that if $B$ is the event that some $X^{(i)}$ in the $k / h$ copies of $X$ in $f_{h}(X)$ takes one of its top values, $\left(x_{1}, \ldots, x_{t}\right)$, then certainly

$$
f_{h}(X \mid B)=\mathbb{E}\left(\max \left(X^{(1)}, \ldots, X^{(k / h)}\right) \mid B\right) \geq f_{h}(X) .
$$

(as we are conditioning on an event concentrated on the highest possible values). But the left-hand side can be written out in full as

$$
\frac{1}{\left(1-\left(1-q_{t}\right)^{k / h}\right)}\left(\left(1-\left(1-q_{1}\right)^{k / h}\right) x_{1}+\cdots+\left(\left(1-q_{t-1}\right)^{k / h}-\left(1-q_{t}\right)^{k / h}\right) x_{t}\right) \geq f_{h}(X) .
$$

But this is just

$$
\frac{1}{\left(1-\left(1-q_{t}\right)^{k / h}\right)} \cdot f_{h}\left(X^{\prime}\right) \geq f_{h}(X) .
$$

Noting that $1-\left(1-q_{t}\right)^{k / h} \geq\left(1-\frac{1}{\sqrt{e}}\right)$ gives the result.

We have therefore shown that a transformation mapping $X$ to $X^{\prime}$, non-zero only on the top $h / 2 k$ quantile of $X$, does not result in too large a loss in the value of $f_{h}(X)$.

Lemma 2.6. Let $X$ have $\left(x_{1}, \ldots, x_{t}\right)$ as its top values, with $q_{t}=\frac{h}{2 k}$. Then

for any $l \geq t$

$$
x_{l}<\frac{f_{h}(X)}{1-\frac{1}{\sqrt{e}}}
$$

Proof. Note that

$$
\left(1-\left(1-q_{t}\right)^{k / h}\right) x_{t} \leq f_{h}(X)
$$

The lemma then follows by noting that $\left(1-\left(1-q_{t}\right)^{k / h}\right)>1-\frac{1}{\sqrt{e}}$, and that $x_{l} \leq x_{t}$ for $l \geq t$.

Next, we prove a simple lemma on certain functions increasing in value and then invoke this lemma to show that, for random variables with total probability mass corresponding to positive values less than $h / 2 k$, we can bound our test function $f_{h}$ with respect to the canonical test of expected value and with respect to a conditional expectation. Again, these lemmas will bound specific parts of bounds relating $f_{h}$ and $g_{h}$.

LEMMA 2.7. For $a \geq 1$, the functions

$$
(1-x)^{a}-(1-a x)
$$

and

are increasing for $x \in\left[0, \frac{1}{2 a}\right]$.

$$
\left(1-\frac{a}{2} x\right)-(1-x)^{a}
$$

Proof. Differentiating, and remo ving the positive factor of $a$, we have

$$
1-(1-x)^{a-1}
$$

which is $\geq 0$ for $x \in[0,1]$ and

$$
(1-x)^{a-1}-\frac{1}{2}
$$

which achieves its minimum value at $x=\frac{1}{2 a}$ but remains non-negative for $a \geq 1$.

LEMMA 2.8. For a random variable $X$, with total probability mass for positive values $\leq h / 2 k$ (i.e., $\left.q_{n} \leq h / 2 k\right)$, we have

$$
\frac{h f_{h}(X)}{k} \leq \mathbb{E}(X) \leq \frac{2 h f_{h}(X)}{k}
$$


Proof. $f_{h}(X)$ can be written explicitly as

$$
\left(1-\left(1-q_{1}\right)^{k / h}\right) x_{1}+\left(\left(1-q_{1}\right)^{k / h}-\left(1-q_{2}\right)^{k / h}\right) x_{2}+\cdots+\left(\left(1-q_{n-1}\right)^{k / h}-\left(1-q_{n}\right)^{k / h}\right) x_{n} .
$$

Noting that $q_{i}<q_{i+1}$, a straightforward application of Lemma 2.7 gives

$$
\frac{k p_{i+1}}{2 h} \leq\left(1-q_{i}\right)^{k / h}-\left(1-q_{i+1}\right)^{k / h} \leq \frac{k}{h} p_{i+1} .
$$

Substituting this into the expression for $f_{h}(X)$ gives

$$
\frac{k \mathbb{E}(X)}{2 h}=\sum_{i=1}^{n} \frac{k p_{i} x_{i}}{2 h} \leq f_{h}(X) \leq \sum_{i=1}^{k} \frac{k p_{i} x_{i}}{h}=\frac{k \mathbb{E}(X)}{h}
$$

Lemma 2.9. For a random variable $X$, underlying sample space $[0,1]$, let $A$ be as in Definition 2.4 . Then

$$
\mathbb{E}(X \mid A) \leq 4 f_{h}(X)
$$

Proof. Splitting the the boundary value $x_{t}$ if necessary, assume $q_{t}=\frac{h}{2 k}$. But then for $X^{\prime}$ as in Lemma 2.5

$$
f_{h}\left(X^{\prime}\right)=\left(1-\left(1-q_{1}\right)^{k / h}\right) x_{1}+\cdots+\left(\left(1-q_{t-1}\right)^{k / h}-\left(1-q_{t}\right)^{k / h}\right) x_{t} \leq f_{h}(X)
$$

As $q_{t}=\frac{h}{2 k}$, we can use Lemma 2.7 (with $a=k / h, q_{i} \in[0, k / 2 h], i \leq t$ ) to get

$$
\frac{k}{2 h} \mathbb{E}\left(X^{\prime}\right) \leq f_{h}\left(X^{\prime}\right) \leq f_{h}(X)
$$

Also

Therefore,

$$
\mathbb{E}(X \mid A)=\frac{1}{q_{t}}\left(\sum_{j=1}^{t} p_{j} x_{j}\right)=\frac{2 k}{h}\left(\sum_{j=1}^{t} p_{j} x_{j}\right)=\frac{2 k}{h} \mathbb{E}\left(X^{\prime}\right) .
$$

$$
\mathbb{E}(X \mid A) \leq 4 f_{h}(X) .
$$

In summary, we've seen that we can bound contributions of the top $h / 2 k$ quantile to $f_{h}$ and upper bound the contribution of the tail. We've also seen that we can upper and lower bound the expectation and the conditional expectation of $X$ using $f_{h}$.

\subsection{A Test with Constant Factor Approximation to Optimal}

Using the preliminary results we proved in the previous section, this section puts them together to give our main result:

THEOREM 2.10. If $X_{1}, \ldots, X_{k}$ are the top scorers for the test function $f_{h}$, and $Y_{1}, \ldots, Y_{k}$ is the true optimal team with respect to the team performance scoring function $g_{h}$, then for constant $\lambda,(\lambda<30)$,

$$
g_{h}\left(Y_{1}, \ldots, Y_{k}\right) \leq \lambda g_{h}\left(X_{1}, \ldots, X_{k}\right) .
$$

The proof proceeds in two steps. First, we show an upper bound for $g_{h}$ in terms of $f_{h}$. In particular, if every member of the team $X_{i}$ has $f_{h}\left(X_{i}\right) \leq c$, we show that the team performance (according to $g_{h}$ ) is $\leq A c$, where $A$ is a constant. After proving a similar lower bound, we can put the two together to get our desired constant factor approximation.

\section{The Upper Bound.}

Theorem 2.11. Let $X_{1}, \ldots, X_{k}$ be random variables with $f_{h}\left(X_{i}\right) \leq c$. Then

$$
g_{h}\left(X_{1}, \ldots, X_{k}\right) \leq 2 h c+\frac{h c}{1-\frac{1}{\sqrt{e}}} .
$$


Proof. Assume the underlying sample space is $[0,1]^{k}$. Let $S \subset[k]$, and

$$
B_{S}=\left\{\omega \in[0,1]^{k}: \omega_{i}>1-\frac{h}{2 k} \Longleftrightarrow i \in S\right\} ;
$$

that is, the event that $X_{i}$ takes values in its top $h / 2 k$ quantile if and only if $i \in S$. For a sample point $\omega \in B_{S}$, note that

$$
\left(X_{X_{1}, \ldots, X_{k}}^{(1)}, \ldots, X_{X_{1}, \ldots, X_{k}}^{(h)}\right)(\omega) \leq \sum_{i \in S} X_{i}(\omega)+\frac{h c}{1-\frac{1}{\sqrt{e}}} .
$$

Indeed, if the top $h$ values are $X_{n_{1}}, \ldots, X_{n_{h}}$, with the first $m, n_{1}, \ldots, n_{m}$ in $S$, then

$$
\sum_{i=1}^{m} X_{n_{i}}(\omega) \leq \sum_{i \in S} X_{i}(\omega)
$$

The remaining random variables, $X_{n_{m+1}}, \ldots, X_{n_{h}}$ take tail values (as in Definition 2.4), so by Lemma 2.6,

$$
\sum_{i=m+1}^{h} X_{n_{i}}(\omega)<(h-m) \frac{c}{1-\frac{1}{\sqrt{e}}} \leq \frac{h c}{1-\frac{1}{\sqrt{e}}}
$$

giving the inequality. Summing up over all $\omega \in B_{S}$, we get

$$
g_{h}\left(\left(X_{1}, \ldots, X_{k}\right) 1_{B_{S}}\right) \leq \mathbb{E}\left(1_{B_{S}} \sum_{i \in S} X_{i}\right)+\mathbb{P}\left(B_{S}\right) \frac{h c}{1-\frac{1}{\sqrt{e}}} .
$$

But letting $A_{i}$ be the event that $\omega_{i}>1-\frac{h}{2 k}$, and using independence of the $X_{i}$ and linearity of expectation

$$
\mathbb{E}\left(1_{B_{S}} \sum_{i \in S} X_{i}\right)=\mathbb{P}\left(B_{S}\right) \sum_{i \in S} \mathbb{E}\left(X_{i} \mid A_{i}\right) .
$$

Using the bound in Lemma 2.9, this becomes

$$
\mathbb{E}\left(1_{B_{S}} \sum_{i \in S} X_{i}\right) \leq \mathbb{P}\left(B_{S}\right)|S| 4 c .
$$

Finally, as $\mathbb{P}\left(B_{S}\right)=\prod_{i \in S} \mathbb{P}\left(A_{i}\right) \prod_{i \notin S}\left(1-\mathbb{P}\left(A_{i}\right)\right)$,

$$
\mathbb{P}\left(B_{S}\right)=\left(\frac{h}{2 k}\right)^{|S|}\left(1-\frac{h}{2 k}\right)^{k-|S|}
$$

that is, the number of $X_{i}$ taking their top values follows a binomial distribution, parameters $\left(k, \frac{h}{2 k}\right)$. So, summing up over $B_{S}$ for all $S \subset[k]$, we get

$$
g_{h}\left(X_{1}, \ldots, X_{k}\right) \leq \sum_{i=0}^{k}\left(\begin{array}{l}
k \\
i
\end{array}\right)\left(\frac{h}{2 k}\right)^{i}\left(1-\frac{h}{2 k}\right)^{k-i} i \cdot 4 c+\frac{h c}{1-\frac{1}{\sqrt{e}}} .
$$

Noting that the first term on the right hand side is just the mean $(h / 2)$ of the binomial distribution scaled by $4 c$ gives the result.

The Lower Bound. We now move on to a lower bound. We first give a lower bound for the case $h=1$, when $g_{h}=\mathbb{E}(\max (\cdot))$, and show how to extend this for general $h$. To prove the $h=1$ case, we will use our transformation in Lemma 2.5 to zero all values lower than the top $1 / 2 k$ quantile and prove a lower bound on random variables with total positive probability mass $\leq 1 / 2 k$. We thus first state and derive this. 
Lemma 2.12. Let $X_{1}, \ldots, X_{k}$ all have total positive probability mass $\leq \frac{1}{2 k}$, with $f_{1}\left(X_{i}\right) \geq c$ for all i. Then

$$
\mathbb{E}\left(\max \left(X_{1}, \ldots, X_{k}\right)\right) \geq 2 c\left(1-\frac{1}{\sqrt{e}}\right)
$$

Proof. For any $X_{i}$, let $A_{i}$ be the event that $X_{i}$ is nonzero. We lower bound the expected maximum as follows: Given $X_{1}, \ldots, X_{k}$ in that order, we output the value of the first nonzero random variable we come across (starting from $X_{1}$ and finishing at $X_{k}$.)

This output value is pointwise less than or equal to the true maximum, so its expected value is a lower bound on the expected maximum. But its expected value is just

$$
\mathbb{P}\left(A_{1}\right) \mathbb{E}\left(X_{1} \mid A_{1}\right)+\left(1-\mathbb{P}\left(A_{1}\right)\right) \mathbb{P}\left(A_{2}\right) \mathbb{E}\left(X_{2} \mid A_{2}\right)+\cdots+\left(\prod_{i=1}^{k-1}\left(1-\mathbb{P}\left(X_{i}\right)\right) \mathbb{E}\left(X_{k}\right) .\right.
$$

Noting that $\mathbb{P}\left(A_{i}\right) \mathbb{E}\left(X_{i} \mid A_{i}\right)=\mathbb{E}\left(X_{i}\right)$ and that $\left(1-\mathbb{P}\left(A_{i}\right)\right) \geq\left(1-\frac{1}{2 k}\right)$, we get

$$
\mathbb{E}\left(\max \left(X_{1}, \ldots, X_{k}\right)\right) \geq \mathbb{E}\left(X_{1}\right)+\left(1-\frac{1}{2 k}\right) \mathbb{E}\left(X_{2}\right)+\cdots+\left(1-\frac{1}{2 k}\right)^{k-1} \mathbb{E}\left(X_{k}\right) .
$$

Using the lower bound of $\mathbb{E}\left(X_{i}\right) \geq \frac{f_{1}\left(X_{i}\right)}{k}$ from Lemma 2.8, summing up the geometric series, and noting $\left(1-\frac{1}{2 k}\right)^{k} \geq\left(1-\frac{1}{\sqrt{e}}\right)$, we have

$$
\mathbb{E}\left(\max \left(X_{1}, \ldots, X_{k}\right)\right) \geq 2 c\left(1-\frac{1}{\sqrt{e}}\right)
$$

as desired.

We now prove our lower bound for $h=1$.

Theorem 2.13. Let $X_{1}, \ldots, X_{k}$ be random variables with $f_{1}\left(X_{i}\right) \geq c$ for all $i$. Then

$$
\mathbb{E}\left(\max \left(X_{1}, \ldots, X_{k}\right)\right) \geq 2 c\left(1-\frac{1}{\sqrt{e}}\right)^{2} .
$$

Proof. For any $X_{i}$ with total positive probability mass $>\frac{1}{2 k}$, we apply the transformation in Lemma 2.5 to get $X_{i}^{\prime}$, which is a lower bound on $X_{i}$. So certainly

$$
\mathbb{E}\left(\max \left(X_{1}, \ldots, X_{k}\right)\right) \geq \mathbb{E}\left(\max \left(X_{1}^{\prime}, \ldots, X_{k}^{\prime}\right)\right),
$$

and, by Lemma 2.5 ,

$$
f\left(X_{i}^{\prime}\right) \geq c\left(1-\frac{1}{\sqrt{e}}\right)
$$

so using Lemma 2.12, the statement of the theorem follows.

We now apply this to prove the main lower bound theorem.

Theorem 2.14. Let $X_{1}, \ldots, X_{k}$ be random variables with $f_{h}\left(X_{i}\right) \geq c$ for all $i$. Then,

$$
g_{h}\left(X_{1}, \ldots, X_{k}\right) \geq 2 h c\left(1-\frac{1}{\sqrt{e}}\right)^{2} .
$$

Proof. Note that, certainly,

$$
g_{h}\left(X_{1}, \ldots, X_{k}\right) \geq \mathbb{E}\left(\max \left(X_{1}, \ldots, X_{k / h}\right)\right)+\cdots+\mathbb{E}\left(\max \left(X_{k-h+1}, \ldots, X_{k}\right)\right) .
$$


But each term on the right-hand side is bounded below by $2 c\left(1-\frac{1}{\sqrt{e}}\right)^{2}$ by using Theorem 2.13. So, summing together, we have

$$
g_{h}\left(X_{1}, \ldots, X_{k}\right) \geq 2 h c\left(1-\frac{1}{\sqrt{e}}\right)^{2}
$$

as desired.

Finishing the Proof. With established lower and upper bounds, Theorem 2.10 follows easily.

Proof (Theorem 2.10). First note that if $l<h$, we can define $g_{h}\left(X_{1}, \ldots, X_{l}\right)$ to be the sum of the expectations of all the $X_{i}$ as this is the same as adding $h-l$ random variables, each deterministically 0 .

Without loss of generality, let $\left\{Y_{1}, \ldots, Y_{k}\right\}=\left\{Y_{1}, \ldots, Y_{l}, X_{l+1}, \ldots, X_{k}\right\}$; that is, $X_{l+1}, \ldots, X_{k}$ is the intersection of the team formed of best test scorers and the optimal team. Now, if $c=$ $\min _{i} f_{h}\left(X_{i}\right)$, then for $j \leq l$, as any $Y_{j}$ is not in the top $k$ scorers, $f_{h}\left(Y_{j}\right) \leq c$.

Note that

$$
2 g_{h}\left(X_{1}, \ldots, X_{k}\right) \geq g_{h}\left(X_{1}, \ldots, X_{k}\right)+g_{h}\left(X_{l+1}, \ldots, X_{k}\right) .
$$

Using the lower bound from Theorem 2.14, we get

$$
2 g_{h}\left(X_{1}, \ldots, X_{k}\right) \geq 2 h c\left(1-\frac{1}{\sqrt{e}}\right)^{2}+g_{h}\left(X_{l+1}, \ldots, X_{k}\right) .
$$

On the other hand,

$$
g_{h}\left(Y_{1}, \ldots, X_{l+1}, \ldots, X_{k}\right) \leq g_{h}\left(Y_{1}, \ldots, Y_{l}\right)+g_{h}\left(X_{l+1}, \ldots, X_{k}\right) .
$$

Using the upper bound from Theorem 2.11 then gives

$$
g_{h}\left(Y_{1}, \ldots, X_{l+1}, \ldots, X_{k}\right) \leq 2 h c+\frac{h c}{1-\frac{1}{\sqrt{e}}}+g_{h}\left(X_{l+1}, \ldots, X_{k}\right) .
$$

So we get that

$$
g_{h}\left(Y_{1}, \ldots, Y_{k}\right) \leq \lambda g_{h}\left(X_{1}, \ldots, X_{k}\right)
$$

where

$$
\lambda=\frac{2\left(1-\frac{1}{\sqrt{e}}\right)+1}{\left(1-\frac{1}{\sqrt{e}}\right)^{3}} .
$$

\subsection{A Different Test}

In the previous section we proved the main result of the article, that there exists a test function, $f_{h}$, evaluating "potential," that can be used to select a team whose performance, according to a team performance function $g_{h}$, is only a constant factor from the optimal, independent of team size.

A natural follow-up question is whether $f_{h}$ is the only such test. From the proof, we can see that this is not the case. If $E=\left\{\omega: \omega>1-\frac{h}{k}\right\}$ for $\omega \in[0,1]$, the underlying sample space, then choosing $X$ according to the value of

$$
\mathbb{E}(X \mid E)
$$

also provides a constant-factor approximation to the optimal set. 
THeORem 2.15. If $X_{1}, \ldots, X_{k}$ are random variables with the $k$ highest values of $\mathbb{E}\left(X_{i} \mid E_{i}\right)$, where $E_{i}$ is the event that $X_{i}$ takes its top $h / k$ quantile of values, and $Y_{1}, \ldots, Y_{k}$ is the optimal set size $k$, then for a constant $\mu$ independent of $k$,

$$
g_{h}\left(Y_{1}, \ldots, Y_{k}\right) \leq \mu g_{h}\left(X_{1}, \ldots, X_{k}\right) .
$$

The two proofs are similar, which is expected, as the analysis of the function $f_{h}(\cdot)$ makes use of quantities derived from $\mathbb{E}(X \mid E)$. The function $f_{h}(\cdot)$ seems the more natural of the two; however, it is arguably more direct to think about testing an individual through repeated independent evaluations than to try quantifying what his or her top $h / k$ values are likely to be. The full proof is included in the Appendix.

\subsection{A Best Approximation?}

In this section, we've seen that there exists a natural individual test, the potential test, that can get to within a constant factor $(\approx 30)$ of optimal. We then outlined a different test (arguably slightly less natural to implement) which also gets to within a constant factor of the optimal $(\approx 16)$.

Seeing these constants, we might ask whether we can say something on whether there is some constant factor $C>1$ which no test can achieve. We prove that such a $C$ does indeed exist.

THEOREM 2.16. No test function $f$ can guarantee a constant factor approximation to the optimal closer than $9 / 8=1.125$ when evaluating team performance with the expected maximum.

Proof. Our proof is with a bad example. Assume we have three weighted Bernoulli random variables $X_{1}, X_{2}, X_{3}$ from which we wish to pick a team of size 2. A weighted Bernoulli random variable is one that takes exactly one nonzero value $v$ with some probability $p$, and can thus be characterized by the vector $(p, v)$.

In that format, let our three Bernoulli random variables be $X_{1}=(1 / 2,2), X_{2}=(1,1), X_{3}=$ $(1 / 2,4 / 3)$. Note that $X_{1}$ is monotonically better than $X_{3}$, so any sensible test function $f$ should definitely pick $X_{1}$ and one of $X_{2}, X_{3}$. Indeed, if the team were to comprise of $\left(X_{2}, X_{3}\right)$, this would result in an expected maximum of $7 / 6$, a factor of $9 / 7$ away from the optimal team's expected maximum of $3 / 2$.

Breaking ties adversarially (as we can always perturb an example slightly in a tie), if $f\left(X_{3}\right)>$ $f\left(X_{2}\right)$, then our team becomes $\left(X_{1}, X_{3}\right)$, but the expected maximum of this team is $4 / 3$, whereas the expected maximum of the team $\left(X_{1}, X_{2}\right)$ is $3 / 2$, and so $f$ is $9 / 8$ from optimal.

If, on the other hand, $f\left(X_{2}\right)>f\left(X_{3}\right)$, then consider a new triple of random variables $Y_{1}=$ $(1,1), Y_{2}=(1,1), Y_{3}=(1 / 2,4 / 3)$. As $Y_{1}, Y_{2}=X_{2}$ and $Y_{3}=X_{3}, f$ will pick the team $\left(Y_{1}, Y_{2}\right)$, which has an expected maximum of 1 compared to picking a team of $\left(Y_{1}, Y_{3}\right)$ where the expected maximum is $7 / 6$, meaning $f$ is $7 / 6$ away from optimal.

So the best any test statistic can manage in this setting is a constant factor approximation of $9 / 8=1.125$.

\section{SUBMODULARITY AND NEGATIVE EXAMPLES}

In this section, we recap properties of submodularity, prove the pointwise submodularity of $g_{h}$, and study the failure of the canonical test. We then more broadly look at submodular functions in general. We show that, among submodular functions, the existence of an individual test function $f_{h}$ which can be used for a proof of constant factor optimality is an uncommon feature relying on the unique properties of the expected maximum. 


\subsection{Submodularity, Pointwise Submodularity and the Canonical Test}

Earlier, we claimed that $\mathbb{E}(\max (\cdot))$ is submodular. In fact, a stronger statement is true. To state it, we recall our notation in which, for a set $T$ of random variables, $X_{T}^{(j)}$ denotes the $j$ th largest in the set.

THeOREM 3.1. Let $\mathcal{U}$ be a large finite ground set of non-negative random variables, with $\Omega$ being the underlying sample space. In a slight abuse of notation, for $\omega \in \Omega$, and $h \geq 0$, let

$$
\omega_{h}: \mathcal{P}(U) \rightarrow \mathbb{R}
$$

be defined by

$$
\omega_{h}(T)=\left(X_{T}^{(1)}+\cdots+X_{T}^{(h)}\right)(\omega)
$$

that is, the sum of the top $h$ values of the random variables in $T$ evaluated at the sample point $\omega$. Then, $\omega_{h}(\cdot)$ is submodular.

In summary, we prove that if $A=S \backslash\{y\}$, with $S \subset \mathcal{U}$, then for $x \notin S$, the submodular property

$$
\omega_{h}(S \cup\{x\})-\omega_{h}(S) \leq \omega_{h}(A \cup\{x\})-\omega_{h}(A)
$$

holds. We show this by fixing an order of elements in $S$ under $\omega$ and considering what each side of the inequality looks like. Chaining a set of inequalities of this form by removing one element each time gives the result for arbitrary subsets of $S$.

(Note that if $|A|<h$, only the first $|A|$ terms are possibly nonzero: We can increase $|A|$ by adding a number of deterministically zero random variables.)

Proof (Theorem 3.1). Assume $S=\left\{X_{1}, \ldots, X_{n}\right\}$, and $A=\left\{X_{1}, \ldots, X_{n-1}\right\}$. Rearranging, the submodularity inequality becomes

$$
\omega\left(A \cup\left\{X, X_{n}\right\}\right)+\omega(A) \leq \omega(A \cup\{X\})+\omega\left(A \cup\left\{X_{n}\right\}\right) .
$$

First note that $X, X_{n}$ are interchangeable in the above inequality. We examine two cases.

(1) At least one of $X, X_{n}$, wlog $X$ (by symmetry) is not in the top $h$ values in $\omega$. This has two easy subcases. If $|A| \geq h$, then

$$
\omega_{h}\left(A \cup\left\{X, X_{n}\right\}\right)+\omega_{h}(A)=\omega_{h}\left(A \cup\left\{X_{n}\right\}\right)+\omega_{h}(A)
$$

and

$$
\omega_{h}(A \cup\{X\})+\omega_{h}\left(A \cup\left\{X_{n}\right\}\right)=\omega_{h}(A)+\omega_{h}\left(A \cup\left\{X_{n}\right\}\right),
$$

so equality holds. In the other case, we have $|A|<h$, so we get

$$
\omega_{h}(A \cup\{X\})+\omega_{h}\left(A \cup\left\{X_{n}\right\}\right)=\omega_{h}(A)+X(\omega)+\omega_{h}(A)+X(\omega) .
$$

The left-hand side of the target inequality becomes

$$
\omega_{h}\left(A \cup\left\{X, X_{n}\right\}\right)+\omega_{h}(A) \leq\left(A+X+X_{n}\right)(\omega)+A(\omega)
$$

with strict inequality if $|A|=h-1$, as $X$ would be omitted in this case. So again, the desired inequality holds.

(2) Now, we may assume that $X_{n}, X$ are both in the top $h$. Assume

$$
X_{n}(\omega)=X_{A \cup\left\{X, X_{n}\right\}}^{(i)},
$$

and

$$
X(\omega)=X_{A \cup\left\{X, X_{n}\right\}}^{(j)},
$$


and wlog $i>j$. In $A \cup\left\{X, X_{n}\right\}$, let the top $h+2$ elements (with appropriately many zero elements) be ordered as below:

$X_{n_{1}}(\omega) \geq X_{n_{2}}(\omega) \geq \ldots X_{n_{i-1}}(\omega) \geq X_{n}(\omega) \geq X_{n_{i+1}}(\omega) \geq \ldots X_{n_{j-1}}(\omega) \geq X(\omega) \geq X_{n_{j+1}}(\omega) \geq \ldots X_{n_{h+2}}(\omega)$.

Then we get

$$
\omega_{h}\left(A \cup\left\{X, X_{n}\right\}\right)+\omega_{h}(A)=\left(2\left(\sum_{\substack{l=1 \\ l \neq i, j}}^{h-2} X_{n_{l}}\right)+X+X_{n}+X_{n_{h+1}}+X_{n_{h+2}}\right)(\omega)
$$

and

$$
\omega_{h}(A \cup\{X\})+\omega_{h}\left(A \cup\left\{X_{n}\right\}\right)=\left(2\left(\sum_{\substack{l=1 \\ l \neq i, j}}^{h-2} X_{n_{l}}\right)+X+X_{n}+2 X_{n_{h+1}}\right)(\omega) .
$$

Noting that $X_{n_{h+1}} \geq X_{n_{h+2}}$ gives the result.

A useful corollary is:

Corollary 3.2. For $h \geq 1, g_{h}(\cdot)$ is submodular.

which follows from the theorem by taking expectations.

There are many results about the tractability (or approximate tractability) of optimization problems associated with submodular functions. For our purposes here, the most useful among these results is the approximate maximization of arbitrary monotone submodular functions over sets of size $k$. This can be achieved by a simple greedy algorithm, which starts with the empty set and, at each stage, iteratively adds the element providing the greatest marginal gain; the result is a provable $(1-1 / e)$ approximation to the true optimum [19]. Note that this means we can find a good approximation of the optimal set even when the random variables $X_{i}$ are dependent. (See Section 4 for further discussion of this.)

The Canonical Test. In Section 2, our motivation for studying $f_{h}$, a measure of potential, was the failure of the canonical test in selecting a team according to $\mathbb{E}(X)$. Here, we use the property of submodular functions to prove the failure of this test.

OBSERvation 1. If $f$ is a submodular function on $\mathcal{P}(U)$, then for every $S \subset U$

$$
f(S) \leq \sum_{x \in S} f(\{x\}) .
$$

This naturally leads to:

Proposition 3.3. If $g_{h}(\cdot)$ is the team evaluation metric, with $Y_{1}, \ldots, Y_{k}$ being the true optimal set, and $X_{1}, \ldots, X_{k}$ the random variables with the $k$ highest expectations (with $\mathbb{E}\left(X_{i}\right) \geq \mathbb{E}\left(X_{j}\right)$ if $i \geq j$ ) then

and this bound is tight.

$$
g_{h}\left(Y_{1}, \ldots, Y_{k}\right) \leq \frac{k}{h} g_{h}\left(X_{1}, \ldots, X_{k}\right),
$$

Proof. By the observation, we note that

$$
g_{h}\left(Y_{1}, \ldots, Y_{k}\right) \leq \sum_{i=1}^{k} g_{h}\left(Y_{i}\right)=\sum_{i=1}^{k} \mathbb{E}\left(Y_{i}\right)
$$


But as $X_{1}, \ldots, X_{k}$ are the elements with the $k$ highest expectations,

$$
\sum_{i=1}^{k} \mathbb{E}\left(Y_{i}\right) \leq \sum_{i=1}^{k} \mathbb{E}\left(X_{i}\right) \leq \frac{k}{h} \sum_{i=1}^{h} \mathbb{E}\left(X_{i}\right)
$$

the last inequality following from the assumption on the ordering of the $X_{i}$. Finally,

$$
g_{h}\left(X_{1}, \ldots, X_{k}\right) \geq g_{h}\left(X_{1}, \ldots, X_{h}\right)=\sum_{i=1}^{h} \mathbb{E}\left(X_{i}\right),
$$

the last equality as there are only $h$ values. Putting it together, we have

$$
g_{h}\left(Y_{1}, \ldots, Y_{k}\right) \leq \frac{k}{h} g_{h}\left(X_{1}, \ldots, X_{h}\right) \leq \frac{k}{h} g_{h}\left(X_{1}, \ldots, X_{k}\right),
$$

as desired. For tightness, let $X_{i}$ be deterministically $1+\epsilon$ and $Y_{i}$ be $n$ with probability $1 / n$ for large $n$. Then

$$
g_{h}\left(Y_{1}, \ldots, Y_{k}\right) \geq \sum_{i=0}^{h} i n\left(\begin{array}{l}
k \\
i
\end{array}\right)\left(\frac{1}{n}\right)^{i}\left(1-\frac{1}{n}\right)^{k-i} \geq n\left(1-\left(1-\frac{1}{n}\right)^{k}\right)=k+O\left(\frac{1}{n}\right) .
$$

Also,

$$
g_{h}\left(X_{1}, \ldots, X_{k}\right)=h(1+\epsilon)
$$

So as $n \rightarrow \infty$ and $\epsilon \rightarrow 0$, we have

$$
g_{h}\left(Y_{1}, \ldots, Y_{k}\right) \rightarrow \frac{k}{h} g_{h}\left(X_{1}, \ldots, X_{k}\right) .
$$

\subsection{Test Scores for Other Submodular Functions}

In the previous section, we saw that for $g=\mathbb{E}(\max (\cdot))$, a submodular function, we were able to define an individual test score with a constant factor approximation to the optimal. Furthermore, we were able to define a family of submodular functions $g_{h}$ interpolating between the expected maximum and a sum of expectations, which all had this property. It is therefore natural to wonder whether this is a property shared by many submodular functions. One way to formalize this question might be the following:

QuEstion 2. Given a (potentially infinite) universe $U$, for which associated submodular functions $g$ does there exist a test score $f$

$$
f: U \rightarrow \mathbb{R}^{+}
$$

such that, for any subset $S \subset U$, if $x_{1}, \ldots, x_{k} \in S$ are the elements with the $k$ highest values of $f$, then $g\left(x_{1}, \ldots, x_{k}\right)$ is always a constant-factor approximation to

$$
\max _{T \subset S,|T|=k} g(T) ?
$$

Despite the positive result in Section 2, we find that many common submodular functions depend too heavily on the interrelations between elements for independent evaluations of elements to work well. We present two such examples.

Cardinality Function. One of the canonical examples of a submodular function is the set cardinality function. Let $U=\mathcal{P}(\mathbb{N})$. Then for $T=\left\{T_{1}, \ldots, T_{m}\right\}$, with $T_{i} \in U$,

$$
g(T)=\left|\cup_{i=1}^{m} T_{i}\right| \text {. }
$$

This function has a natural interpretation for team performance. We can imagine each candidate as a set $T_{i}$, consisting of the set of perspectives they bring to the task. $g\left(T_{1}, T_{2}, \ldots, T_{m}\right)$ is then 
the total number of distinct perspectives that the team members bring collectively; this objective function is used in arguments that diverse teams can be more effective [11,17].

We show a negative result for the use of test scores with this function.

THEOREM 3.4. In the above setting, with universe $U$ and $g$ the set cardinality function, no such test score $f$ exists.

Proof. Suppose for contradiction such an $f$ did exist. Assume ties are broken in the worst way possible (no information is gained from a tie.) Let $U_{1}, U_{2}, \ldots$ be disjoint intervals in $\mathbb{N}$ with

$$
U_{i}=\{(i-1)(k+1)+1, \ldots, i(k+1)\} .
$$

And let

$$
V_{i}=\left\{S \subset U_{i}:|S|=k\right\}
$$

that is, the set of all size $k$ subsets of $U_{i}$. We will find it useful to label elements of $V_{i}$ based on their $f$ value, so let

$$
V_{i}=\left\{X_{i 1}, \ldots, X_{i k+1}\right\}
$$

with

$$
f\left(X_{i 1}\right) \leq f\left(X_{i 2}\right) \ldots \leq f\left(X_{i k+1}\right) .
$$

Call a set $V_{j}, j>k$ bad with respect to $V_{1}$ if

$$
f\left(X_{j 1}\right) \leq f\left(X_{12}\right)
$$

and good otherwise. Note that we cannot have more than $k V_{j}$ bad with respect to $V_{1}$. Else, supposing $V_{n_{1}}, \ldots, V_{n_{k}}$ were all bad with respect to $V_{1}$, in the set

$$
S=\left\{X_{12}, \ldots, X_{1 k+1}, X_{n_{1} 1}, \ldots, X_{n_{k} 1}\right\},
$$

the $k$ set chosen by $f$ would be $X_{12}, \ldots, X_{1 k+1}$, for a $g$ value of $k+1$, but the optimum is given by $X_{n_{1} 1}, \ldots, X_{n_{k} 1}$, for a $g$ value of $k^{2}-$ a factor of $\approx k$ difference.

So there are at most $k$ bad sets with respect to $V_{1}$. But the same logic applies to $V_{2}, \ldots, V_{k}$. So, in $V_{k+1}, \ldots, V_{k^{2}+k+1}$, there is at least one set, say $V_{j}$, that is good with respect to $V_{1}, \ldots, V_{k}$. But then in the set

$$
S=\left\{X_{11}, \ldots, X_{k 1}, X_{j 1}, \ldots, X_{j k}\right\}
$$

the $k$ set chosen by $f$ would be $X_{j 1}, \ldots, X_{j k}$, with a $g$ value of $k+1$, but the optimum would be $X_{11}, \ldots, X_{k 1}$ with a $g$ value of $k^{2}$.

Linear Matroid Rank Functions. Another class of measures of team performance is given by assigning each candidate a vector $v_{i} \in \mathbb{R}^{m}$, and the performance of a team $v_{1}, v_{2}, \ldots, v_{k}$ is the rank of the span of the set of corresponding vectors. Such a measure has a similar motivation to the previous set cardinality example: If the team is trying to solve a classification problem over a multidimensional feature space, then $v_{i}$ may represent the weighted combination of features that candidate $i$ brings to the problem, and the span of $v_{1}, v_{2}, \ldots, v_{k}$ establishes the effective number of distinct dimensions the team will be able to use.

More generally, the rank of the span of a set of vectors is a matroid rank function, and we can ask the question in that context. Given a matroid $(V, \mathcal{I})$ and a set $S \subset V$, the matroid rank function $g$ is

$$
g(S)=\max \{|T|: T \subset S, T \in \mathcal{I}\}
$$

that is, the maximal independent set contained in $S$. It is well known that matroid rank functions are submodular [2]. To come back to our vector space example, we show that when our underlying set is $\mathbb{R}^{m}$ and $\mathcal{I}$ are subsets that are linearly independent, no single element test can capture the relation between vectors well. 
Theorem 3.5. For $U, g$ as above, no test score with good approximation exists.

The proof of this theorem relies on the fundamental property of $\mathbb{R}$. We show that, for any sequence along a specific direction, the $f$ values for this sequence must be bounded. By the defining property of $\mathbb{R}$, each sequence then has a convergent subsequence. Looking at these convergent subsequences along each of $k$ coordinate axes $e_{1}, \ldots, e_{k}$, we can then pick our bad set, fooling $f$ into choosing $O(k)$ points in the same direction. See the Appendix for a full proof.

\subsection{Result for a Supermodular Function}

The preceding two examples show bad cases for submodular functions. As is expected, supermodular functions also have a negative answer to Question 3.5.

A classic example of a supermodular function is the edge count function.

Definition 3.6. Given a graph $G=(V, E)$ and a set $S \subset V, g(S)$ is the number of edges in the induced subgraph with vertex set $S$.

It is easy to check that $g$ is supermodular. $g$ also forms our bad example for supermodular functions.

THEOREM 3.7. Let $U$ be a very large graph containing at least $N$ disjoint complete graphs with $k+1$ vertices (i.e., $K_{k+1}$ ). Then there is no test score $f$ with a constant (independent of $k$ ) order approximation property to the optimal $k$ set with respect to $g$.

The proof is very similar to the cardinality function case. In that, we wanted to avoid picking subsets of the same set; in this, we would like to pick as many vertices in a single clique as possible. We adjust the notion of bad accordingly to ensure this doesn't happen, and we arrive at our desired contradiction identically to before.

A particularly interesting feature of this case is that, without the canonical statistical test for submodular functions, we can have an arbitrarily bad approximation ratio; even if $f$ is defined to be constant on each vertex, the counterexample demonstrates that $f$ may pick a set with no induced edges.

\section{HILL CLIMBING AND OPTIMALITY}

For most nontrivial submodular functions, finding the optimal solution is computationally intractable. This is the case for the maximum of a set of random variables that are not necessarily independent. In particular, suppose that $S=\left\{X_{1}, X_{2}, \ldots, X_{n}\right\}$ is a set of dependent random variables. For a set $T$ of them, we can define $g(T)$ to be the expected maximum of the random variables in $T$. We now argue that maximizing $g(T)$ is an NP-hard problem in general. We will do this by reducing an instance of Set Cover to the problem.

Recall that in set cover, we have a universe $U$ and a set $T=\left\{S_{1}, \ldots, S_{n}\right\}$ of subsets of $U$; that is, $S_{i} \subset U$ for all $i$. We wish to know if there is a subset $T^{\prime} \subset T$, with $\left|T^{\prime}\right| \leq k$, such that $\bigcup_{S_{i} \in T^{\prime}} S_{i}=U$. To model this with random variables, let the underlying sample space be $U$ and each $X_{i}=1_{S_{i}}$ the indicator function for the set $S_{i}$. Then it is easy to see that there exists a team size $k$ with expected maximum 1 if and only if there exists $T^{\prime}$ as above, $\left|T^{\prime}\right| \leq k$. So, maximizing the expected maximum of a set size $k$ provides an answer to the NP complete decision problem.

In terms of approximation, we can apply the general hill-climbing result mentioned earlier [19] to provide a $(1-1 / e)$ approximation for finding the set of $k$ dependent random variables with the largest expected maximum.

A natural question is whether independence is a strong enough assumption to guarantee a better approximation ratio. Indeed, we may even be tempted to ask the following: 
Question 3. If $X_{1}, \ldots, X_{n}$ are (discrete) independent random variables, does hill-climbing find the size $k$ set maximizing the expected maximum?

Unfortunately, the answer is no. For a simple counterexample, take $X$ taking positive values $(9 / 5,6 / 5)$ with respective probability masses $(1 / 3,1 / 3), Y$ deterministically $1+\epsilon$ for $\epsilon$ very small, and $Z$ taking a positive value $3 / 2$ with probability $2 / 3$. Then $\mathbb{E}(Y)>\mathbb{E}(X), \mathbb{E}(Z)$, which means that, in the first step, hill-climbing would choose $Y$. But,

$$
\mathbb{E}(\max (X, Z))>\mathbb{E}(\max (Y, Z)), \mathbb{E}(\max (X, Y)),
$$

so hill-climbing would not find the optimal solution. In this counterexample, $Y, Z$ are both examples of weighted Bernoulli random variables.

Definition 4.1. We say a random variable $X$ has the weighted Bernoulli distribution if $X=x$ for some $x \geq 0$ with probability $p$, and $X=0$ otherwise.

What is surprising is that when all our random variables are weighted Bernoulli, Question 3 has an affirmative answer.

THEOREM 4.2. Given a pool of random variables, each of weighted Bernoulli distribution, performing hill-climbing with respect to $\mathbb{E}(\max (\cdot))$ finds the size $k$ set maximizing the expected maximum.

In the context of forming teams, we can think of candidates with weighted Bernoulli distributions as having a sharply "on-off" success pattern: They have a single way to succeed, producing a given utility, and otherwise they provide zero utility.

For $X$ as above, we will find it convenient to denote $X$ as $(p, x)$. For two weighted Bernoulli random variables $X=(p, x)$ and $Y=(q, y)$, we use $X \geq Y$ to mean $x \geq y$. For $X_{i}=\left(p_{i}, x_{i}\right)$, with $X_{1} \geq . . \geq X_{k}$, the expected maximum has an especially clean form:

$$
\mathbb{E}\left(\max \left(X_{1}, \ldots, X_{k}\right)\right)=p_{1} x_{1}+\left(1-p_{1}\right) p_{2} x_{2}+\ldots \prod_{i=1}^{k-1} p_{k} x_{k} .
$$

Rewriting this slightly, it also has an intrinsically recursive structure

$$
\mathbb{E}\left(\max \left(X_{1}, \ldots, X_{k}\right)\right)=p_{1} x_{1}+\left(1-p_{1}\right) \mathbb{E}\left(\max \left(X_{2}, \ldots, X_{k}\right)\right) .
$$

As a step toward proving Theorem 4.2, we need two useful lemmas describing when random variables can be exchanged without negatively affecting the expected maximum. Assume from now on that all random variables are weighted Bernoulli.

Our first lemma shows that if one random variable dominates another in both nonzero value and expectation, we may always substitute in the dominating variable. So, given two random variables with the same expected value, we always prefer the "riskier" random variable.

Lemma 4.3. If $X \geq Y$, and $\mathbb{E}(X) \geq \mathbb{E}(Y)$, then for any $X_{1}, \ldots, X_{k}$,

$$
\mathbb{E}\left(\max \left(X, X_{1}, \ldots, X_{k}\right)\right) \geq \mathbb{E}\left(\max \left(Y, X_{1}, \ldots, X_{k}\right)\right) .
$$

Proof (Lemma 4.3). Assume $X_{i}$ are in value order. Without loss of generality, assume $X \geq X_{i}$ for all $i$ (an almost identical proof works if that is not the case) and that $X_{t} \geq Y \geq X_{t+1}$. Letting $X=(p, x), X_{i}=\left(p_{i}, x_{i}\right)$. Also, assume that $Y=(q, y)$. By the recursive structure of the expected maximum for weighted Bernoulli random variables,

$$
\mathbb{E}\left(\max \left(X, X_{1}, \ldots, X_{k}\right)\right)=p x+(1-p) b+(1-p) s c
$$

and that

$$
\mathbb{E}\left(\max \left(Y, X_{1}, \ldots, X_{k}\right)\right)=b+s q y+s(1-q) c
$$


where

$$
\begin{aligned}
b & =\mathbb{E}\left(\max \left(X_{1}, \ldots, X_{t}\right)\right) \\
s & =\mathbb{P}\left(X_{1}, \ldots, X_{t}=0\right) \\
c & =\mathbb{E}\left(\max \left(X_{t+1}, \ldots, X_{k}\right)\right) .
\end{aligned}
$$

Note $b+s c \leq x$ as $X \geq X_{1}, \ldots, X_{k}$. So, if $p \geq q$,

$$
p x+(1-p)(b+s c) \geq q x+(1-q)(b+s c)
$$

The left-hand side of the above is just $\mathbb{E}\left(\max \left(X, X_{1}, \ldots, X_{k}\right)\right)$, so we can assume $p \leq q$ by decreasing $p$ to $q$ if necessary, and this will only decrease the value of $\mathbb{E}\left(\max \left(X, X_{1}, \ldots, X_{k}\right)\right)$. Now, note that

$$
\mathbb{E}\left(\max \left(X, X_{1}, \ldots, X_{k}\right)\right) \geq \mathbb{E}\left(\max \left(Y, X_{1}, \ldots, X_{k}\right)\right) \Longleftrightarrow p x-p b-s q y+(q-p) s c \geq 0 .
$$

But $b /(1-s)$ is a convex combination of $X_{1}, \ldots, X_{t}$, so $b /(1-s) \leq x$. So,

$$
p x-p b-s q y+(q-p) s c \geq s p x-s q y+(q-p) s c .
$$

Finally, by assumption, $\mathbb{E}(X) \geq \mathbb{E}(Y)$, and $p \leq q$, so the result holds.

The next lemma describes a slightly technical variant of the preceding substitution rule.

Lemma 4.4. Let $X \geq Y$, and $\mathbb{E}\left(\max \left(X, X_{1}, \ldots, X_{k}\right)\right) \geq \mathbb{E}\left(\max \left(Y, X_{1}, \ldots, X_{k}\right)\right)$. Then, if $Y_{1}, \ldots, Y_{m}$ such that $Y \geq Y_{i}$ for all $i$,

$$
\mathbb{E}\left(\max \left(X, X_{1}, \ldots, X_{k}, Y_{1}, \ldots, Y_{m}\right)\right) \geq \mathbb{E}\left(\max \left(Y, X_{1}, \ldots, X_{k}, Y_{1}, \ldots, Y_{m}\right)\right) .
$$

The proof of this lemma is similar to the first lemma and is in the Appendix.

We can now easily prove Theorem 4.2 .

Proof (Theorem 4.2). We prove this inductively, showing that the element chosen by hillclimbing at time $i$ is part of the optimal set from then on. Our base case is proving the first element chosen, $X=(x, p)$, which has greatest expectation, is always in the optimal set. Suppose the optimal set size $k$ is $\left\{Y_{1}, \ldots, Y_{k}\right\}$. Then, if some $Y_{i} \leq X$, by Lemma 4.3, we could replace $Y_{i}$ by $X$. So $X \leq Y_{k}$. But as $Y_{k}$ only appears as $\mathbb{E}\left(Y_{k}\right)$ in $\mathbb{E}\left(\max \left(Y_{1}, \ldots, Y_{k}\right)\right.$, and $X$ has greatest expectation, we can replace $Y_{k}$ by $X$.

Suppose we have chosen $t$ random variables, $X_{1} \geq \ldots \geq X_{t}$, with the th random variable chosen being $X_{i}$. By the induction hypothesis, we know $X_{j}$ for $j \neq i$ are part of any $\geq t$ sized optimal set. For an optimal solution size $k$, let $Y_{1} \geq \ldots \geq Y_{m}$ (where $m$ may equal 0 ) be the random variables distinct from $X_{i}$, between $X_{i-1}$ and $X_{i+1}$ value-wise. Similarly, let $Z_{1} \geq \ldots \geq Z_{h}$ be the random variables between $X_{i+1}$ and $X_{k}$. We have a few cases.

First note if $m>0$ and $X_{i} \geq Y_{j}$ some $j$, then as $\mathbb{E}\left(\max \left(X_{i}, \ldots, X_{t}\right)\right) \geq \mathbb{E}\left(\max \left(Y_{j}, X_{i+1}, \ldots, X_{t}\right)\right)$, by applying Lemma 4.4 , we can swap $Y_{j}$ with $X_{i}$. So, $X_{i} \leq Y_{j}$ for all $j$, or $m=0$. In either case, if $h>0$, applying Lemma 4.4 again, we may swap $X_{i}$ with $Z_{1}$. So, $h=0$; and so, in order value, the final string of random variables in the optimal set is just $X_{i}, X_{i+1}, \ldots, X_{k}$. Note that if we take the smallest random variable distinct from the $X_{l}$ larger than $X_{i}$, say $Y, X_{j} \geq Y \geq X_{j+1}$, then, as

$$
\mathbb{E}\left(\max \left(X_{1}, \ldots, X_{t}\right)\right) \geq \mathbb{E}\left(\max \left(Y, X_{1}, \ldots, X_{i-1}, X_{i+1}, \ldots X_{t}\right)\right)
$$

from the choice of elements by the hill-climbing algorithm, by the recursive structure of the expected maximum, we must have

$$
\mathbb{E}\left(\max \left(X_{j}, X_{j+1}, \ldots, X_{i}, \ldots, X_{t}\right)\right) \geq \mathbb{E}\left(\max \left(X_{j}, Y, \ldots, X_{i-1}, X_{i+1}, \ldots, X_{t}\right)\right),
$$

so we can swap $Y$ with $X_{i}$. This completes the induction step and the proof. 
This proof method gives us a simple condition which is sufficient (though slightly stronger than necessary) for when the hill-climbing algorithm finds the optimal set.

Condition 4. Let $f$ be a submodular function on a universe $U$. If $S_{t}=\left\{x_{1}, \ldots, x_{t}\right\}$ is the set picked by hill-climbing at time $t$, (with $S=\emptyset)$ at $t=0$, and $x_{t+1}$ is the next element chosen by hill-climbing, then, for any $Z \subset U \backslash S_{t}$, we must have

$$
\max _{z \in Z} f\left(S_{t} \cup\left\{x_{t+1}\right\} \cup Z \backslash\{z\}\right) \geq f\left(S_{t} \cup Z\right) .
$$

For submodular functions satisfying Condition 4, it is possible to prove the optimality of hillclimbing as above. Given that $S_{t}$ is part of the optimal set, we show that we can always substitute in $x_{t+1}$ into the optimal solution and ensure that the value of $f$ doesn't decrease. Hence, $x_{t+1}$ must be part of the optimal set.

\section{TEST SCORES FOR COMPETITION}

Thus far, we have considered a setting in which we want to assemble a collaborative team, and we use test scores to identify team members. But there are other natural contexts where we can ask about the power of fixed "scores" to identify the quality of participants, and one of these is a setting in which there is competition between individuals.

There is a large literature on the use of numerical scores to represent the quality of participants in a competitive domain (e.g., $[6,10])$. Our purpose in this short section is to describe a basic result establishing a tight limit on the power of such scores in an abstract setting.

We consider the following simple model of competition between pairs of individuals. Each possible competitor $i$ in our setting is represented by a random variable $X_{i}$; we can think of $X_{i}$ as representing the distribution of how well $i$ will perform in any given competition. Thus, when competitors $i$ and $j$ are paired against each other, each draws independently from her respective random variables $X_{i}$ and $X_{j}$; these draws represent their performance in this instance of the $i-j$ competition. The competitor who draws the larger number is the winner. (If they draw equal values, we declare them to have tied.)

Now, by analogy with previous sections-but adapted here to our competitive setting-we would like to assign a numerical score to each competitor so that by comparing the scores of $i$ and $j$, we can form an estimate of which is likely to win in a competition between them.

A natural question is whether we can find a score for each competitor so that the competitor with the higher score in a pairwise competition is more likely to win. Formulating this to allow for the possibility of ties as well, we'd like a function $f$ that maps random variables to real numbers, so that if $X_{i}$ and $X_{j}$ are random variables with $f\left(X_{i}\right) \geq f\left(X_{j}\right)$ then

$$
\mathbb{P}\left(X_{i} \geq X_{j}\right) \geq \frac{1}{2}
$$

It turns out that such a function does not exist. To establish this fact, we use a counterintuitive probabilistic structure known as nontransitive dice. A set of nontransitive dice is a collection of random variables $X_{1}, \ldots, X_{n}$ for which $\mathbb{P}\left(X_{i}>X_{i+1}\right)>1 / 2$ (with addition taken modulo $n$, so that $\mathbb{P}\left(X_{n}>X_{1}\right)>1 / 2$ as well).

Here is a simple example, using six-sided dice $X, Y, Z$ with nonstandard sets of numbers written on their six faces. Suppose

- $X$ has sides 2, 2, 4, 4, 9, 9;

- $Y$ has sides $1,1,6,6,8,8$;

- $Z$ has sides $3,3,5,5,7,7$. 
Then it is easy to compute that

$$
\mathbb{P}(X>Y)=\mathbb{P}(Y>Z)=\mathbb{P}(Z>X)=\frac{5}{9}
$$

It is known that for all $\gamma<3 / 4$, there exist sets of nontransitive dice $X_{1}, \ldots, X_{n}$ for which $\mathbb{P}\left(X_{i}>\right.$ $\left.X_{i+1}\right)>\gamma[16,21,22]$.

Using nontransitive dice, one can directly put a limit on the power of test scores for competition.

THeOREM 5.1. Let $f$ be any function mapping random variables to real numbers, and let $\beta>1 / 4$. Then there exist random variables $X$ and $Y$ for which $f(X) \geq f(Y)$ but $\mathbb{P}(X \geq Y)<\beta$.

Proof. Since $1-\beta<3 / 4$, we can find a set of nontransitive dice $X_{1}, \ldots, X_{n}$ for which $\mathbb{P}\left(X_{i}>\right.$ $\left.X_{i+1}\right)>1-\beta$. For any function $f$ mapping random variables to real numbers, let us apply $f$ to each of $X_{1}, \ldots, X_{n}$. Let $f\left(X_{i}\right)$ be a maximum value among $f\left(X_{1}\right), \ldots, f\left(X_{n}\right)$. Then we have $f\left(X_{i}\right) \geq f\left(X_{i-1}\right)$ (since $f\left(X_{i}\right)$ is a maximum value), but $\mathbb{P}\left(X_{i-1}>X_{i}\right)>1-\beta$ by the definition of the sequence of nontransitive dice; and hence $\mathbb{P}\left(X_{i} \geq X_{i-1}\right)<\beta$.

Let us state this result in slightly different language. A test score is any function $f$ mapping random variables to real numbers. We say that $f$ has resolution $\alpha$ if, for all random variables $X$ and $Y$ with $f(X) \geq f(Y)$, we have $\mathbb{P}(X \geq Y) \geq \alpha$. Then Theorem 5.1 shows that there is no test score with resolution $1 / 2$, and in fact no test score with resolution $\alpha$ for any $\alpha>1 / 4$.

Suppose, then, that we were to weaken our goal and simply ask: Is there a test score with some positive resolution $\alpha>0$ ? We now show, via a simple construction, that this is the case: In fact, there is a test score with resolution $1 / 4$, establishing that the negative result of Theorem 5.1 is tight.

Theorem 5.2. Let $f$ be a function that maps a random variable $X$ to $a$ median value; that is, $a$ number $x$ such that $\mathbb{P}(X \geq x) \geq 1 / 2$ and $\mathbb{P}(X \leq x) \geq 1 / 2$. (Note that such an $x$ need not be unique.)

Then, if $X$ and $Y$ are random variables with $f(X) \geq f(Y)$, we have $\mathbb{P}(X \geq Y) \geq 1 / 4$. That is, $f$ is a test score with resolution $1 / 4$.

Proof. The proof follows directly from the definition of a median value. Suppose $f(X) \geq f(Y)$. Then

$$
\mathbb{P}(X \geq Y) \geq \mathbb{P}(X \geq f(X)) \mathbb{P}\left(Y \leq f(Y) \geq \frac{1}{2} \cdot \frac{1}{2}=\frac{1}{4} .\right.
$$

\section{CONCLUSION AND OPEN PROBLEMS}

In this article, we have demonstrated that, for a natural family of submodular performance metrics, team selection can happen solely on an individual basis, with minimal concession in team quality. However, this selection criterion is more intricate than the canonical test (singleton set value), the performance of which we also characterized. Not all submodular functions are amenable to such an approximation, and we exhibited examples where no function could always guarantee a constant order bound. This leads to the natural question of whether it is possible to characterize the truly submodular functions (functions for which, like the expected maximum, the canonical test performs poorly) which can approximated in such a fashion. There may be an opportunity to connect such questions to a distinct literature on approximating a submodular function with only a small number of values known [8] and approximation by juntas [7]. Another interesting direction is to relax the assumption of knowing the distribution of our random variables $X_{i}$. In many real-life scenarios, we may not have a true skill distribution for candidates but may instead have to rely on noisy samples. This problem may have links to work on robust estimation [12].

Finally, we also explored the implications of independence of random variables when using hillclimbing to approximate the size- $k$ set maximizing the expected maximum. We established that, 
for certain random variables, we could find the true optimum this way. A natural question is, then, for what distributional assumptions can we guarantee optimality or a significantly better approximation ratio? Much work has been done on structural properties of ensembles of random variables with different distributions [3, 4], and it is possible that such techniques may be useful here.

\section{A APPENDIX}

Here we provide a proof of 2.15 .

Proof. Note that if we find upper and lower bounds like Theorem 2.11 and Theorem 2.14, then we can use the final part of the proof of Theorem 2.10 unchanged to give our desired result.

First, note that if $\mathbb{E}(X \mid E) \leq c$, then any value of $X$ not in its top $h / k$ quantile must be $\leq c$ (conditioning on $E$ ensures that the expectation of $X$ is a linear combination of the top values of $X$.) Now, if $X_{1}, \ldots, X_{k}$ such that $\mathbb{E}\left(X_{i} \mid E_{i}\right) \leq c$ for all $i$, then letting $T \subset[k]$ and

$$
C_{T}=\left\{\omega \in[0,1]^{k}: \omega_{i}>1-\frac{h}{k} \Longleftrightarrow i \in T\right\}
$$

be defined analogously to before, we get

$$
g_{h}\left(\left(X_{1}, \ldots, X_{k}\right) 1_{C_{T}}\right) \leq \mathbb{P}\left(C_{T}\right)|T| c+\mathbb{P}\left(C_{T}\right) h c
$$

as before. Summing up we note

$$
\mathbb{P}\left(C_{T}\right)=\left(\frac{h}{k}\right)^{|T|}\left(1-\frac{h}{k}\right)^{k-|T|}
$$

so we have binomial distribution parameters $(k, h / k)$, similar to before, so

$$
g_{h}\left(X_{1}, \ldots, X_{k}\right) \leq \sum_{i=0}^{k} i c \cdot\left(\begin{array}{l}
k \\
i
\end{array}\right)\left(\frac{h}{k}\right)^{i}\left(1-\frac{h}{k}\right)^{k-i}+h c=2 h c .
$$

This gives us an upper bound. The lower bound is of a similar flavor to the upper bound. Suppose $X_{1}, \ldots X_{k}$ such that $\mathbb{E}\left(X_{i} \mid E_{i}\right) \geq c$ for all $i$, and $T$ and $C_{T}$ are as above. Then note that

$$
g_{h}\left(\left(X_{1}, \ldots, X_{k}\right) 1_{C_{T}}\right) \geq \mathbb{P}\left(C_{T}\right) \cdot \min (|T|, h) c ;
$$

that is, for an event $\omega \in C_{T}, g_{h}\left(X_{1}, \ldots, X_{k}\right)$ is greater than summing the minimum of $h$ and $|T|$ of the random variables that take values in their top $h / k$ quantile. Noting that we have the same binomial distribution as before

$$
g_{h}\left(X_{1}, \ldots, X_{k}\right) \geq \sum_{i=h / 2}^{k} \frac{h c}{2} \cdot\left(\begin{array}{l}
k \\
i
\end{array}\right)\left(\frac{h}{k}\right)^{i}\left(1-\frac{h}{k}\right)^{k-i} \geq \frac{h c}{4}
$$

where the last inequality follows by noting that, as the mean of this distribution is $h$, the median is certainly contained in the range $h / 2 \leq i \leq k$.

Note that to be entirely precise, we should replace $h / 2$ with $\left\lfloor\frac{h}{2}\right\rfloor$. The $h=1$ case then needs to be dealt with separately. For $h=1$, note that the probability that at least one of the $X_{i}$ takes a value in its top $h / k=1 / k$ quantile is

$$
1-\left(1-\frac{1}{k}\right)^{k} \geq 1-\frac{1}{e}
$$

So, for the $h=1$ case, we can bound below by

$$
\left(1-\frac{1}{e}\right) c
$$

We finish using the same proof as in Theorem 2.10, getting $\mu=16$. 


\section{A.1 Submodularity and Negative Examples: Proofs}

We first give a proof of Theorem 3.1

Below is the full proof of Theorem 3.5

Proof (Theorem 3.5). As before, we assume for contradiction that such an $f$ does exist. We need a Lemma.

Lemma A.1. Let $x \in \mathbb{R}^{m}$. Then the set

$$
\{f(\lambda x): \lambda \in \mathbb{R}\}
$$

is bounded.

Proof. Suppose not; then there is a sequence $\left(\lambda_{n}\right)_{n \in \mathbb{N}}$ such that

$$
f\left(\lambda_{n} x\right) \geq n \text {. }
$$

But letting $e_{1}, \ldots, e_{k}$ be the standard basis vectors, and $c=\max f\left(e_{i}\right)$, there are $\lambda_{n_{1}}, \ldots, \lambda_{n_{k}}$ with

$$
f\left(\lambda_{n_{i}} x\right)>c,
$$

so in the set $\left\{e_{1}, \ldots, e_{k}, \lambda_{n_{1}} x, \ldots, \lambda_{n_{k}} x\right\}$, the optimal set has rank $k$, but the highest scoring $k$ set has rank 1 .

The consequence (from the fundamental property of the real numbers) is that any sequence of vectors along a particular direction has a convergent subsequence. In particular, defining

$$
a_{i n}=f\left(\frac{e_{i}}{n}\right)
$$

we see that, for each $i,\left(a_{i n}\right)$ has a convergent subsequence. Relabeling if necessary, let this convergent subsequence be $\left(a_{i n}\right)$, with

$$
a_{\text {in }} \rightarrow b_{i}
$$

for each $i$. Without loss of generality, we assume that $b_{1} \geq b_{2} \ldots \geq b_{k}$. We now complete the theorem by examining a few cases.

Case 1: $b_{1}>b_{k / 2}$. In this case, we can take terms very close to $b_{1}$ and terms very close to $b_{i}$ for $i \geq k / 2$ to ensure that we pick all the $a_{1 m}$ terms which only have rank 1 .

In more detail, let $\delta<b_{1}-b_{k / 2}$. Then, as we have a finite number of convergent sequences, $\exists N$ such that for all $m>N,\left|a_{i m}-b_{i}\right|<\delta / 3$ for all $i$. So for $l, m>N$, and for all $i \geq k / 2$ we have

$$
a_{1 m}>a_{i l}
$$

In particular, in the set

$$
\left\{a_{1 m}, \ldots, a_{1(m+k)}, a_{(k / 2) l}, \ldots, a_{k l}\right\}
$$

the $k$ set with the maximum $f$ values are the first $k$, for a rank of 1 , but the optimal set can achieve rank $k / 2+1$ (taking say the last $k / 2+1$ elements), providing the desired contradiction.

Case 2: $b_{1}=\cdots=b_{k / 2}=b$. Here, we derive a contradiction by looking more closely at what each sequence $a_{i j}$ for $i \leq k / 2$ can do and deriving a contradiction. Assume from now on that $i \leq k / 2$.

(i) If for some $i$, say $i=1$, there was $n_{1}, \ldots n_{k}$ and $\delta>0$ such that $a_{1 n_{j}}>b+\delta$, then for $j \neq 1$, picking $a_{j l_{j}}$ within $\delta / 2$ of $b$ would mean $\left\{a_{1 n_{r}}: r \leq k\right\} \cup\left\{a_{j l_{j}}: j \leq k / 2\right\}$ would form a bad set for $f$, with a $2 / k$ approximation ratio. 
(ii) So certainly only finitely many terms $>b$ for any $i$. Discarding them, assume the sequences $a_{i j} \leq b$ for all $i, j$. If for some $i$, say $i=1, k$ or more terms were equal to $b$, say $a_{1 n_{1}}, \ldots, a_{1 n_{k}}$ then for any $j$ (noting we break ties as in the worst case), $f$ performs poorly (2/k approximation) on the set $\left\{a_{1 n_{1}}, \ldots, a_{1 n_{k}}\right\} \cup\left\{a_{21}, \ldots, a_{(k / 2) 1}\right\}$.

(iii) So for each $i$, only finitely many terms $=b$. Discarding those, assume all $a_{i j}<b$. Let $c=$ $\min _{i} a_{i 1}$. Then picking $n_{1}, \ldots, n_{k}$ so $a_{1 n_{k}}>c, f$ has the same poor $2 / k$ approximation on $\left\{a_{11}, \ldots, a_{(k / 2) 1}, a_{1 n_{1}}, \ldots, a_{1 n_{k}}\right\}$.

This completes the proof of the Theorem.

We now give the full proof for the bad example for supermodular functions.

Proof (Theorem 3.7). Assume such an $f$ does exist. Let $K^{1}, \ldots, K^{N}$ be the set of size- $(k+1)$ complete graphs. Let the vertices of $K^{j}$ be $\left\{v_{j 1}, \ldots, v_{j(k+1)}\right\}$ in increasing order of $f$-value, Consider $K^{1}$. For $j>k$, say $K^{j}$ is $b a d$ with respect to $K^{1}$ if $f\left(v_{j(k+1)}\right) \geq f\left(v_{1 k}\right)$. If $K^{n_{1}}, \ldots, K^{n_{k}}$ are all bad with respect to $K^{1}$, then in the set $\left\{v_{11}, \ldots, v_{1 k}, v_{n_{1}(k+1)}, \ldots, v_{n_{k}(k+1)}\right\}$, the set chosen by the test score would be $v_{n_{1}(k+1)}, \ldots, v_{n_{k}(k+1)}$, for no induced edges, while the optimal set is $v 11, \ldots, v_{1 k}$ with $k(k-1) / 2$ induced edges.

So there are less than $k$ graphs bad with respect to $K^{1}$. Similar to the earlier case, applying the same argument to $K^{2}, \ldots, K^{k}$, we note that in $K^{k+1}, \ldots, K^{k^{2}+k+1}$, there is at least one graph that is not bad with respect to all of $K^{1}, \ldots K^{k}$, say $K^{m}$. But then, taking the set $\left\{v_{1(k+1)}, \ldots, v_{k(k+1)}, v_{m 1}, \ldots, v_{m k}\right\}$, the test score pick $v_{1(k+1)}, \ldots, v_{k(k+1)}$ again with no induced edges, while the optimal set is $v_{m 1}, \ldots, v_{m k}$ with $k(k-1) / 2$ edges.

\section{A.2 Hill-Climbing and Optimality}

Given here is the proof of the second lemma to show optimality in the weighted Bernoulli case.

Proof (Lemma 4.4). We prove this by contradiction. Again, we may assume that $X \geq X_{i}$ for all $i, X_{i}$ are in value order, and $X_{t} \geq Y \geq X_{t+1}$ as before. Using the notation of Lemma 4.3, first note that $p \leq q$, as otherwise, $\mathbb{E}(X) \geq \mathbb{E}(Y)$, and we could directly apply Lemma 4.3. Our assumption gives the following inequality:

$$
p x+(1-p) b+(1-p) s c \geq b+s q y+(1-q) s c .
$$

Suppose the Lemma is false. Then, we have

$$
p x+(1-p) b+(1-p) s d<b+s q y+(1-q) s d
$$

where

$$
d=\mathbb{E}\left(\max \left(X_{t+1}, \ldots, X_{k}, Y_{1}, \ldots, Y_{m}\right)\right) .
$$

We show that both of these inequalities cannot hold simultaneously.

As $p \leq q$, we have that

$$
\mathbb{E}\left(\max \left(X, X_{1}, \ldots, Y_{m}\right)\right)-\mathbb{E}\left(\max \left(X, X_{1}, \ldots, X_{k}\right)\right)=(1-p) s(d-c) \geq(1-q) s(d-c) .
$$

But

$$
\mathbb{E}\left(\max \left(Y, X_{1}, \ldots, Y_{m}\right)\right)-\mathbb{E}\left(\max \left(Y, X_{1}, \ldots, X_{k}\right)\right)=(1-q) s(d-c),
$$

Writing

$$
\begin{aligned}
\mathbb{E}\left(\max \left(X, X_{1}, \ldots, Y_{m}\right)\right)= & \left(\mathbb{E}\left(\max \left(X, X_{1}, \ldots, Y_{m}\right)\right)-\mathbb{E}\left(\max \left(X, X_{1}, \ldots, X_{k}\right)\right)\right) \\
& +\mathbb{E}\left(\max \left(X, X_{1}, \ldots, X_{k}\right)\right)
\end{aligned}
$$

and $\mathbb{E}\left(\max \left(Y, X_{1}, \ldots, Y_{m}\right)\right)$ analogously and comparing contradicts the falsity of the Lemma. 


\section{REFERENCES}

[1] C. Ballester, A. Calvó-Armengol, and Y. Zenou. 2006. Who's who in networks. wanted: The key player. Econometrica 74, 5 (2006), 1403-1417.

[2] G. Birkhoff. 1933. On the combination of subalgebras. Cambridge Philosophical Society 29, (1933), 441-464.

[3] C. Daskalakis, I. Diakonikolas, and R. A. Servedio. 2012a. Learning k-modal distributions via testing. In Proceedings of the ACM-SIAM Symposium on Discrete Algorithms. SIAM, Kyoto, Japan, 1371-1385.

[4] C. Daskalakis, I. Diakonikolas, and R. A. Servedio. 2012b. Learning Poisson binomial distributions. In Proceedings of the ACM Symposium on Theory of Computing. 709-728.

[5] H. A. David and H. N. Nagaraja. 2003. Order Statistics (3rd edition). Wiley.

[6] A. Elo. 1978. The Rating of Chess Players, Past and Present. Ishi Press.

[7] V. Feldman and J. Vondrak. 2013. Optimal bounds on approximation of submodular and xos functions by juntas. In Proceedings of the IEEE Symposium on Foundations of Computer Science. 227-236.

[8] M. X. Goemans, N. J. A. Harvey, S. Iwata, and V. Mirrokni. 2009. Approximating submodular functions everywhere. In Proceedings of the ACM-SIAM Symposium on Discrete Algorithms. 535-544.

[9] S. M. Gully, A. Joshi, K. A. Incalcaterra, and J. M. Beaubien. 2002. A meta-analysis of team-efficacy, potency, and performance: Interdependence and level of analysis as moderators of observed relationships. fournal of Applied Psychology 87, 5 (2002), 819-832.

[10] R. Herbrich, T. Minka, and T. Graepel. 2006. Trueskill ${ }^{\mathrm{tm}}$ : A bayesian skill rating system. In Proceedings of the 19th Advances in Neural Information Processing Systems. 569-576.

[11] L. Hong and S. E. Page. 2004. Groups of diverse problem solvers can outperform groups of high-ability problem solvers. Proceedings of the National Academy of Science of the USA 101, 46 (2004), 16385-16398.

[12] P. Huber. 1964. Robust estimation of a location parameter. Annals of Mathematical Statistics 35, 1 (1964), 73-101.

[13] L. B. Jeppesen and K. R. Lakhani. 2010. Marginality and problem-solving effectiveness in broadcast search. Organization Science 21, 5 (2010), 1016-1033.

[14] S. W. J. Kozlowski and D. R. Ilgen. 2006. Enhancing the effectiveness of work groups and teams. Psychological Science in the Public Interest 7, 3 (2006), 77-124.

[15] K. R. Lakhani, K. J. Boudreau, P.-R. Loh, L. Backstrom, C. Baldwin, E. Lonstein, M. Lydon, A. MacCormack, R. A. Arnaout, and E. C. Guinan. 2013. Prize-based contests can provide solutions to computational biology problems. Nature Biotechnology 31, 2 (2013), 108-111.

[16] C. Li-Chien. 1961. On the maximum probability of cyclic random inequalities. Scientia Sinica 10, (1961), 490-504.

[17] L. S. Marcolino, A. X. Jiang, and M. Tambe. 2013. Multi-agent team formation: Diversity beats strength? In Proceedings of the 23rd International foint Conference on Artificial Intelligence.

[18] D. L. Miller. 2001. Reexamining teamwork ksas and team performance. Small Group Research 32, 6 (2001), 745-766.

[19] G. L. Nemhauser and L. A. Wolsey. 1978. Best algorithms for approximating the maximum of a submodular set function. Mathematical Operators Research 3, 3 (1978), 177-188.

[20] S. E. Page. 2008. The Difference: How the Power of Diversity Creates Better Groups, Firms, Schools, and Societies. Princeton University Press.

[21] S. Trybula. 1965. On the paradox of n random variables. Zastos. Mat. 8, (1965), 143-154.

[22] Z. Usiskin. 1964. Max-min probabilities in the voting paradox. Annals of Mathematical Statistics 35, 2 (1964), $857-862$.

[23] S. Wuchty, B. F. Jones, and B. Uzzi. 2007. The increasing dominance of teams in production of knowledge. Science 316, 5827 (2007), 1036-1039.

Received January 2017; revised February 2018; accepted March 2018 